Subscriber access provided by lowa State University | Library

\title{
Article
}

\section{Structure-based design of potent tumor-associated antigens: modulation of peptide presentation by single atom O/S or O/Se substitutions at the glycosidic linkage}

Ismael Compañón, Ana Guerreiro, Vincenzo Mangini, Jorge Castro-López, Margarita Escudero-Casao, Alberto Avenoza, Jesús H. Busto, Sergio Castillón, Jesús Jiménez-Barbero, Juan L. Asensio, Gonzalo Jiménez-Osés, Omar Boutureira, Jesús M. Peregrina, Ramon Hurtado-Guerrero, Roberto Fiammengo, Gonçalo J.L. Bernardes, and Francisco Corzana

J. Am. Chem. Soc., Just Accepted Manuscript • Publication Date (Web): 06 Feb 2019

Downloaded from http://pubs.acs.org on February 6, 2019

\section{Just Accepted}

"Just Accepted" manuscripts have been peer-reviewed and accepted for publication. They are posted online prior to technical editing, formatting for publication and author proofing. The American Chemical Society provides "Just Accepted" as a service to the research community to expedite the dissemination of scientific material as soon as possible after acceptance. "Just Accepted" manuscripts appear in full in PDF format accompanied by an HTML abstract. "Just Accepted" manuscripts have been fully peer reviewed, but should not be considered the official version of record. They are citable by the Digital Object Identifier (DOI®). "Just Accepted" is an optional service offered to authors. Therefore, the "Just Accepted" Web site may not include all articles that will be published in the journal. After a manuscript is technically edited and formatted, it will be removed from the "Just Accepted" Web site and published as an ASAP article. Note that technical editing may introduce minor changes to the manuscript text and/or graphics which could affect content, and all legal disclaimers and ethical guidelines that apply to the journal pertain. ACS cannot be held responsible for errors or consequences arising from the use of information contained in these "Just Accepted" manuscripts. 


\section{Structure-based design of potent tumor-associated antigens: modulation of peptide presentation by single atom $\mathrm{O} / \mathrm{S}$ or $\mathrm{O} / \mathrm{Se}$ substitutions at the glycosidic linkage}

Ismael Compañón, ${ }^{1, \ddagger}$ Ana Guerreiro, ${ }^{2, \ddagger}$ Vincenzo Mangini, ${ }^{3, \ddagger}$ Jorge Castro-López, ${ }^{4}$, Margarita Escudero-Casao, ${ }^{5}$ Alberto Avenoza, ${ }^{1}$ Jesús H. Busto, ${ }^{1}$ Sergio Castillón, ${ }^{5}$ Jesús Jiménez-Barbero, ${ }^{6,7,8}$ Juan L. Asensio, ${ }^{9}$ Gonzalo Jiménez-Osés, ${ }^{1,6,7}$ Omar Boutureira, ${ }^{5}$ Jesús M. Peregrina, ${ }^{1}$ Ramón Hurtado-Guerrero, ${ }^{4}$ Roberto Fiammengo, $3,{ }^{3, *}$ Gonçalo J. L. Bernardes,,$^{2,10, *}$ and Francisco Corzana ${ }^{1, *}$

1 Departamento de Química, Universidad de La Rioja, Centro de Investigación en Síntesis Química, 26006 Logroño, Spain.

2 Instituto de Medicina Molecular, Faculdade de Medicina, Universidade de Lisboa, Avenida Professor Egas Moniz, 1649-028 Lisboa, Portugal.

${ }^{3}$ Center for Biomolecular Nanotechnologies@UniLe, Istituto Italiano di Tecnologia (IIT), 73010 Arnesano, Lecce, Italy.

4 Institute of Biocomputation and Physics of Complex Systems (BIFI), University of Zaragoza, BIFI-IQFR (CSIC), Zaragoza, Spain; Fundación ARAID, 50018, Zaragoza, Spain.

5 Departament de Química Analítica i Química Orgànica, Facultat de Química, Universitat Rovira i Virgili, 43007 Tarragona, Spain.

${ }^{6} \mathrm{CIC}$ bioGUNE, Bizkaia Technology Park, Building 801A, 48170 Derio, Spain.

7 Ikerbasque, Basque Foundation for Science, Maria Diaz de Haro 13, 48009 Bilbao, Spain.

${ }^{8}$ Department of Organic Chemistry II, Faculty of Science \& Technology, University of the Basque Country, 48940 Leioa, Spain.

${ }^{9}$ Instituto de Química Orgánica General, IQOG-CSIC. 28006 Madrid.

10 Department of Chemistry, University of Cambridge, Lensfield Road, CB2 1EW Cambridge, UK.

¥These authors have contributed equally.

Correspondence should be addressed to F.C., G.J.L.B. or R.F.:

francisco.corzana@unirioja.es;

gb453@cam.ac.uk; gbernardes@medicina.ulisboa.pt;

roberto.fiammengo@iit.it 


\begin{abstract}
GalNAc-glycopeptides derived from mucin MUC1 are an important class of tumorassociated antigens. $\alpha$-O-glycosylation forces the peptide to adopt an extended conformation in solution, which is far from the structure observed in complexes with a model anti-MUC1 antibody. Herein, we propose a new strategy to design potent antigen mimics based on modulating peptide/carbohydrate interactions by means of $\mathrm{O} \rightarrow \mathrm{S} / \mathrm{Se}$ replacement at the glycosidic linkage. These minimal chemical modifications bring about two key structural changes to the glycopeptide. They increase the carbohydrate-peptide distance and change the orientation and dynamics of the glycosidic linkage. As a result, the peptide acquires a preorganized and optimal structure suited for antibody binding. Accordingly, these new glycopeptides display improved binding towards a representative anti-MUC1 antibody relative to the native antigens. To prove the potential of these glycopeptides as tumor-associated MUC1 antigen mimics, the derivative bearing the $S$ glycosidic linkage was conjugated to gold nanoparticles and tested as an immunogenic formulation in mice without any adjuvant, that resulted in a significant humoral immune response. Importantly, the mice antisera recognize cancer cells in biopsies of breastcancer patients with high selectivity. This finding demonstrates that the antibodies elicited against the mimetic antigen indeed recognize the naturally occurring antigen in its physiological context. Clinically, the exploitation of tumor-associated antigen mimics may contribute to the development of cancer vaccines and to the improvement of cancer diagnosis based on anti-MUC1 antibodies. The methodology presented here is of general interest for applications because it may be extended to modulate the affinity of biologically relevant glycopeptides towards their receptors.
\end{abstract}




\section{Introduction}

MUC1 mucin is an O-glycoprotein overexpressed in many tumor tissues. ${ }^{1-4}$ Although in healthy cells the backbone of this protein is decorated with complex glycans, in cancer cells this backbone carries rather simple and truncated oligosaccharides. Consequently, different tumor-associated carbohydrate antigens, such as the Tn determinant $(\alpha-O$ GalNAc-Ser/Thr), ${ }^{5}$ are presented to the immune system and can be identified by antiMUC1 antibodies. The peptide fragment Ala-Pro-Asp-Thr-Arg-Pro, which includes the immunodominant PDTRP region of MUC1 tandem repeats, ${ }^{6}$ constitutes the minimum epitope recognized by these antibodies. ${ }^{7}$ Partially glycosylated MUC1 derivatives have been used to prepare immunogenic formulations towards the development of therapeutic cancer vaccines.-12 Similarly, unnatural glycopeptides that mimic tumor-associated MUC1 can find application as biosensors for the detection of cancerous cells. ${ }^{13}$ An intriguing observation about the structural characteristics of these peptides is that $\alpha-O$ glycosylation with GalNAc forces the underlying peptide into an extended conformation in solution as a result of stabilizing interactions, which include direct ${ }^{14}$ or water-mediated hydrogen bonds, between the peptide and the carbohydrate moiety (Figure 1A).15-17 In contrast, the X-ray structure of the glycopeptide epitope bound to an anti-MUC1 antibody (SM3) ${ }^{18}$ revealed a folded conformation around the glycosylated Thr (Figure 1A)..${ }^{19}$ In this case, the sugar shifts the structure of the peptide in solution away from that adopted upon antibody binding. This conformational entropic penalty is however compensated for by favorable enthalpic contributions (hydrogen bonds and $\mathrm{CH} / \pi$ stabilizing interactions) $)^{20,21}$ between the sugar moiety and the antibody. As a result, a modest net increase in binding affinity (around 3-fold) is observed for the glycosylated versus the non-glycosylated peptide.

Herein, we propose a rational approach based on single-atom substitution $(\mathrm{O} \rightarrow \mathrm{S} / \mathrm{Se})$ at the glycosidic linkage to obtain potent antigens with an improved affinity towards antiMUC1 antibodies (Figure 1B). This simple modification increases the distance between the sugar and the peptide fragment - sulfur (or selenium) is larger than oxygen — which in turn minimizes the exo-anomeric effect ${ }^{22}$ and alters the flexibility and the most stable conformation of the glycosidic linkage towards the one optimized for the antibody. Overall, these glycopeptides adopt a distinct structure in solution, which differs markedly from their oxygenated counterparts, thus avoiding the subsequent entropic cost associated to the extended-to-folded conformational transition of the O-glycopeptide in the bound state. In this work, we describe the strong binding of these glycopeptides to a model MUC1 antibody and demonstrate the possibility of using them as tumorassociated MUC1 mimics when they are incorporated in immunogenic formulations. In 
fact, the antibodies elicited in mice recognize selectively the natural occurring tumorassociated MUC1 epitopes displayed on cancer cells in biopsies of breast-cancer patients.

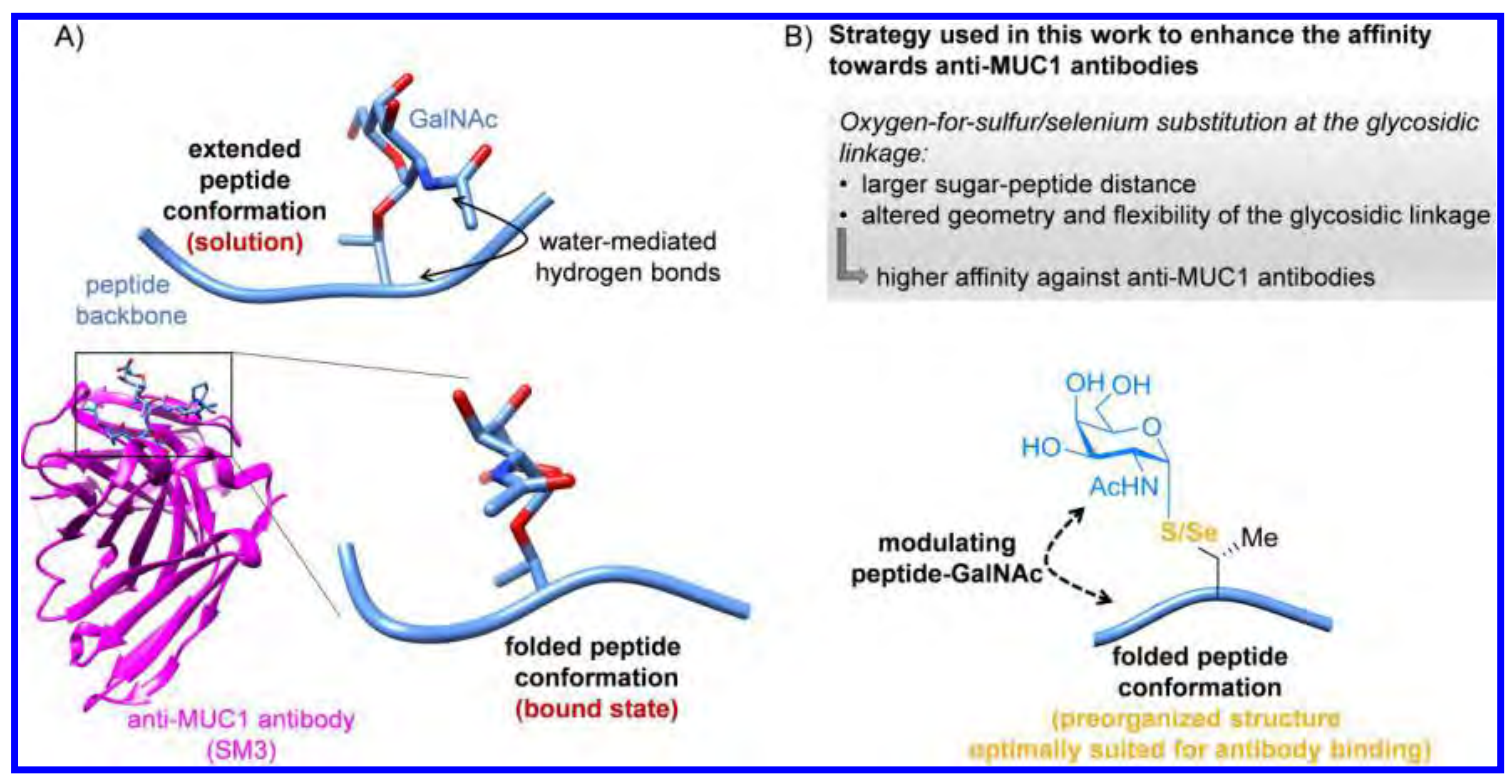

Figure 1. (A) Major conformation of a tumor-associated MUC1 glycopeptide in solution (top) and bound to the SM3 antibody (bottom). (B) Proposed strategy to allow the peptide backbone to adopt a preorganized structure in solution.

\section{Results and Discussion}

\section{Synthesis and conformational analysis of the unnatural glycopeptides in solution}

Glycopeptides $2^{*}$ and $3^{*}$ (Figure $\left.2 A\right)$ were designed to feature $S$ - $(\alpha-D-G a l N A c)$ thiothreonine $\left(\boldsymbol{S T h r}^{\star}\right)$ or $\mathrm{Se}-(\alpha-\mathrm{D}-\mathrm{GaINAc})$-selenothreonine $\left(\mathrm{SeTh}^{\star}\right)$, respectively, as the fourth residue of the Ala-Pro-Asp-Xaa-Arg-Pro epitope. Naturally occurring glycopeptide $1^{\star}$, which has a threonine residue at this position, and non-glycosylated variants 1 and $\mathbf{2}$ were prepared for comparison. 


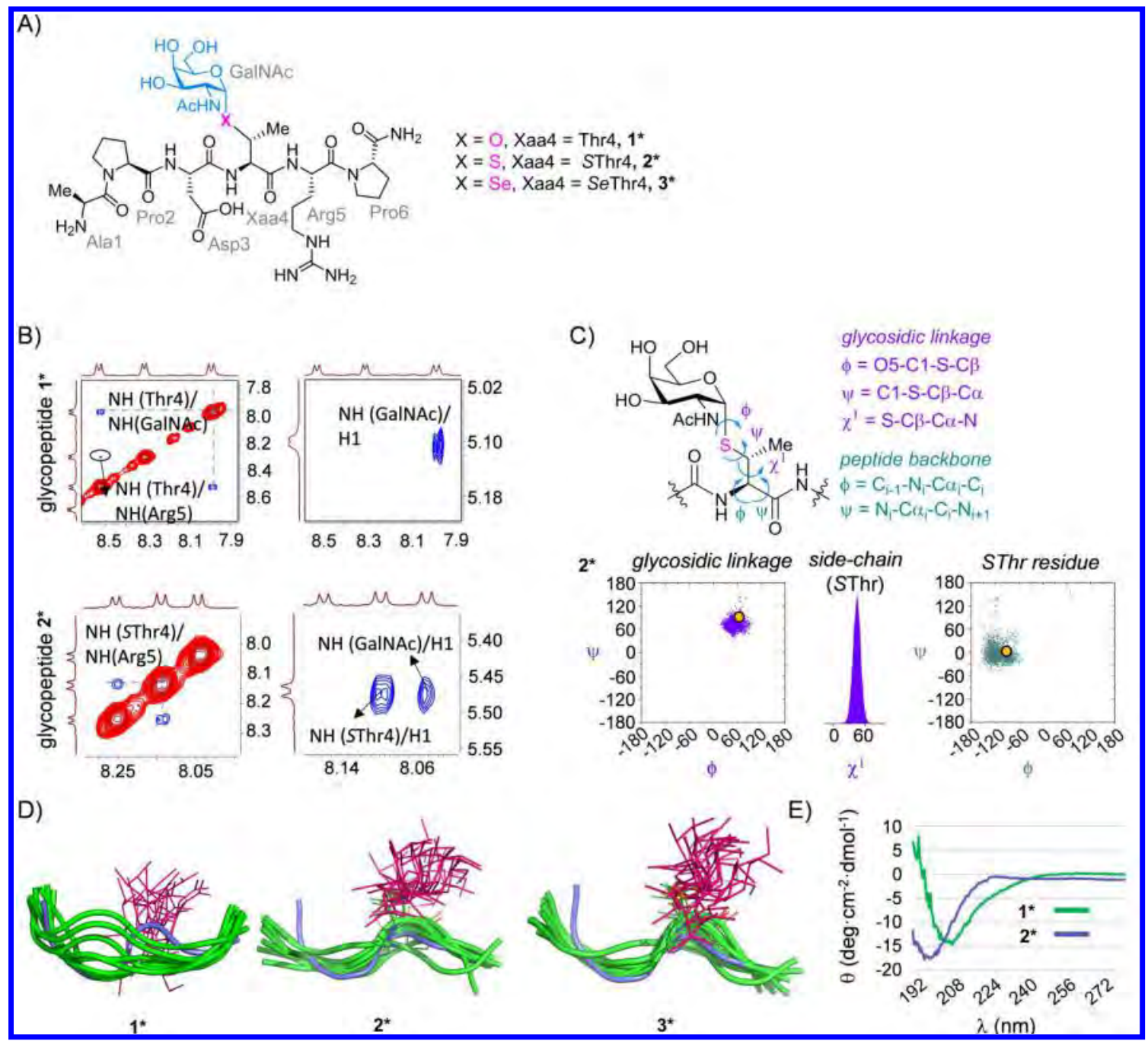

Figure 2. (A) Glycopeptides synthesized and studied in this work, comprising the minimum epitope recognized by most anti-MUC1 antibodies. ${ }^{7}$ (B) Sections of the $500 \mathrm{~ms}$ 2D-ROESY spectrum (400 MHz) in $\mathrm{H}_{2} \mathrm{O} / \mathrm{D}_{2} \mathrm{O}(9: 1)$ at $298 \mathrm{~K}$ and $\mathrm{pH}=6.5$ of glycopeptides $\mathbf{1}^{*}$ (upper panel) and $\mathbf{2}^{*}$ (lower panel) that show the amide region. Diagonal peaks are in red. ROE contacts are represented as blue cross-peaks. A second set of signals is observed corresponding to the cis configuration of the amide bond of proline residues. ${ }^{23}$ (C) Geometry and flexibility at the glycosidic linkage and peptide backbone for the unnatural SThr residue of glycopeptide $2^{*}$ in solution derived from 20 ns experimentguided MD simulations. The yellow circles correspond to the conformation found in the crystal structure of glycopeptide $1^{*}$ bound to a single-chain variable fragment of the SM3 antibody (scFv-SM3, PDB ID: 5A2K). (D) Structural ensembles derived from 20 ns experiment-guided MD simulations for compounds $1^{*}, 2^{*}$ and $3^{*}$ in solution, together with the conformation of the peptide backbone of $1^{*}$ (in blue) found by X-ray crystallography bound to scFv-SM3 antibody (PDB ID: 5A2K). (E) Circular dichroism (CD) spectra of compounds $1^{*}$ and $2^{\star}\left(0.25 \mathrm{mM}\right.$ in sodium phosphate buffer, $\left.\mathrm{pH}=7.5,20^{\circ} \mathrm{C}\right)$. 
Although the synthesis of the amino acid thiothreonine (SThr) has been previously described, ${ }^{24-26}$ the preparation of conveniently protected thiothreonine and selenothreonine derivatives, as well as glycopeptides $\mathbf{2}^{\star}$ and $\mathbf{3}^{\star}$, has not been reported yet. As an example, the synthesis of building block 4 , which is ready to be used in solid phase peptide synthesis (SPPS), is shown in Scheme 1. Conveniently protected threonine 5 was reacted with triphenylphosphine and iodine with imidazole as a base to afford the iodo-derivative 6 with total inversion of configuration at the $\beta$-carbon. ${ }^{27}$ In parallel, selenosugar 9 was prepared in two steps from peracetylated compound 7. In the first step, $\mathbf{7}$ was treated with Woollin's reagent and pyridine to give selenazoline $\mathbf{8}$ in $70 \%$ yield. Hydrolysis of $\mathbf{8}$ with trifluoroacetic acid (TFA) in water afforded selenosugar 9 in moderate yield and as a dimer because of the formation of a diselenide bond. The key step in the synthesis of building block 4 is the nucleophilic attack of $\mathbf{9}$, previously reduced in situ with sodium borohydride, at iodo-derivative 6 . This reaction proceeded in $51 \%$ yield and with total inversion of the configuration at the $\beta$-carbon of the selenothreonine surrogate and completely preserved the $\alpha$-configuration at the anomeric carbon as determined by ${ }^{1} \mathrm{H}$ NMR spectroscopy (see Synthesis section in Supporting Information). Subsequent deprotection steps gave the desired compound $\mathbf{4}$ in $42 \%$ overall yield from $\mathbf{9}$. A similar strategy was used to prepare the building block of SThr * (see Synthesis section in Supporting Information).
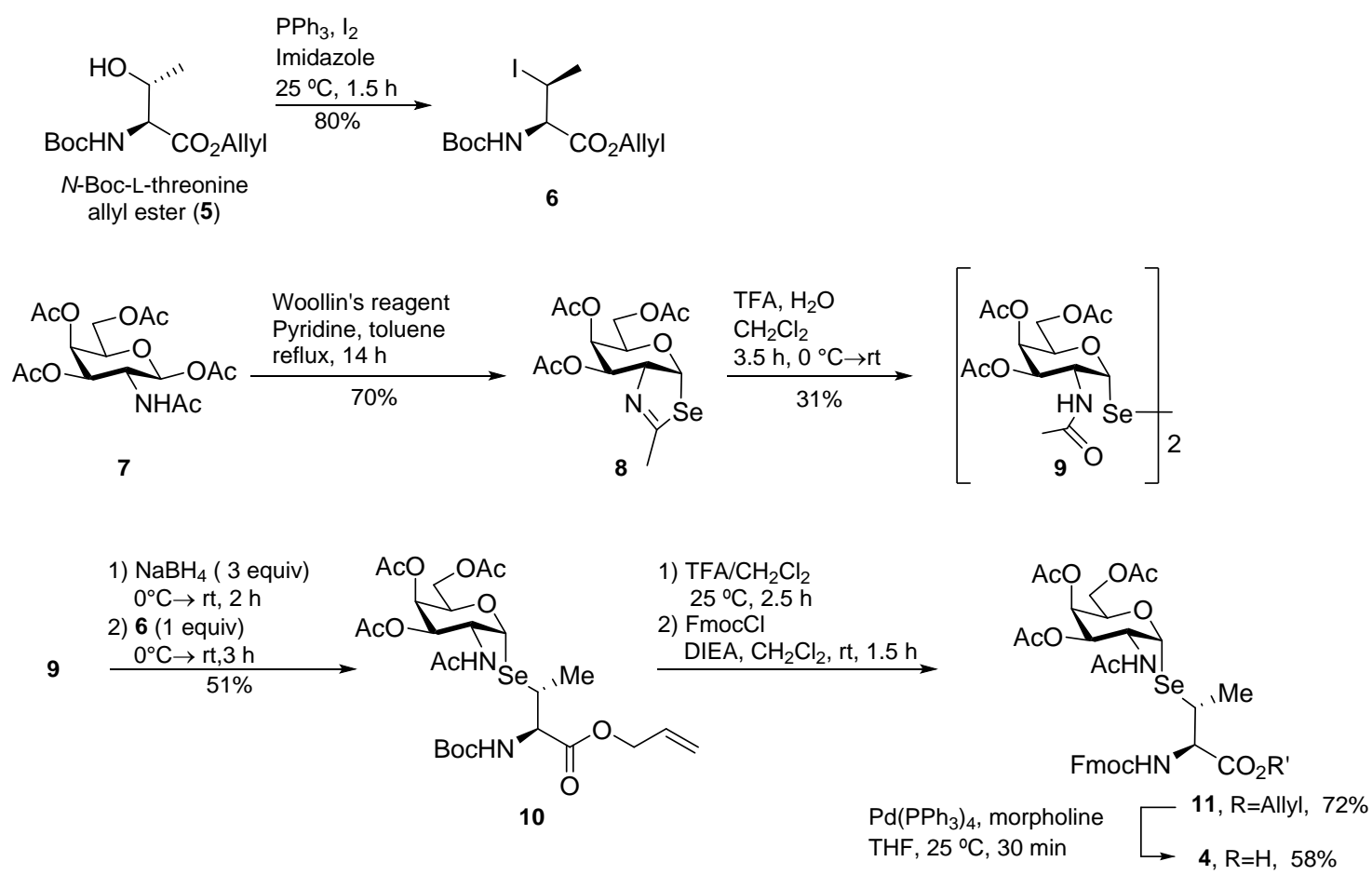

Scheme 1. Synthetic route followed for the preparation of building block 4 . Boc =tertbutyloxycarbonyl. $\mathrm{DIEA}=\mathrm{N}, \mathrm{N}$-diisopropylethylamine. Fmoc=fluorenylmethoxycarbonyl. 
All (glyco)peptides were synthesized with microwave-assisted SPPS (MW-SPPS) by following our reported protocol'13 (Supporting Information). Next, we performed a thorough conformational analysis of the unnatural glycopeptides $\mathbf{2}^{*}$ and $\mathbf{3}^{*}$ in solution, by combining NMR spectroscopic measurements with molecular dynamics (MD) simulations (Figures 2B, 2C and 2D; see also Figures S1 and S2). The lack of a ROESY cross-peak between the $\mathrm{NH}$ of the unnatural SThr4 residue (or SeThr4) and the $\mathrm{NH}$ of GalNAc (Figure 2B, left panel), characteristic of the eclipsed conformation of the glycosidic linkage in GalNAc-Thr, ${ }^{16,17}$ together with the presence of a cross-peak between the NH of SThr4 (or SeThr4) and H1 of GalNAc (Figure 2B, right panel), suggest a different conformation for the $S$ - and Se-containing glycosidic linkages in $\mathbf{2}^{*}$ and $3^{\star}$, respectively, with regard to GalNAc-Thr (Figure 2B). Clear structural differences between glycopeptides $1^{*}$ and the two surrogates $2^{*}$ and $3^{*}$ are also observable in their peptide backbone. In particular, the sequential NH-NH ROESY cross-peak that connects residues 4 and 5 in compounds $2^{*}$ and $3^{*}$ hints to a folded conformation of the glycosylated SThr and SeThr residues (Figure 2B, left panel). ${ }^{28}$ The relevant protonproton distances for the conformational analysis derived from the ROESY spectrum of each compound were then used as time-averaged restraints ${ }^{29,30}$ in experiment-guided MD simulations in accordance with our well-established protocol. ${ }^{31,32}$ The good agreement between the experimental and theoretical derived distances validates our calculations (Table S1 and Figures S4 and S5). According to the MD simulations, the $S$ and Se-glycosidic linkages of $2^{*}$ and $3^{*}$ display a unique conformation centered at $\phi / \psi \approx$ $65^{\circ} / 70^{\circ}$, which agrees with the exo-anomeric effect ${ }^{22}$ and deviates for the more eclipsed arrangement observed for $1^{*}\left(\phi / \psi \approx 65^{\circ} / 120^{\circ}\right){ }^{16}$ (Figure $2 \mathrm{C}$ and Figure S6). It is important to note that this conformer lies at one of the local minima calculated for methyl 4-thio- $\alpha$ maltoside ${ }^{33}$ and it is similar to that explored by an unnatural Tn antigen with a cysteine residue previously prepared in our lab. ${ }^{34}$ The side chain of the unnatural residues in $\mathbf{2}^{*}$ and $3^{*}$ is rather rigid in solution, with conformers characterized by $\chi^{1}=60^{\circ}$. The slightly different geometry and flexibility of $S$ - and $S e$-glycosidic linkages relative to the $O$ glycosidic linkage, together with the larger size of the $S$ and $S e$ atoms, precludes an effective interaction between the peptide backbone and the carbohydrate. In fact, neither significant hydrogen bonds nor water pockets were observed between these moieties. This finding emphasizes the synergistic roles of the methyl group of the threonine and the glycosidic oxygen atom in defining the conformational preference of the natural TnThr antigen.

Regarding the peptide backbone, compounds $2^{*}$ and $3^{*}$ showed conformations characterized by a folded structure around the unnatural residues SThr4 or SeThr4, 
respectively (Figures 2, S4 and S5). This arrangement of the peptide differs from that previously reported for $\mathbf{1}^{*}$ (Figure 2D), which displays mostly a $\beta$-like extended conformation in solution (Figure 2D) owing to water-mediated hydrogen bonds between the peptide and GalNAc. 17,32 The different arrangement of the backbone was also supported by the CD spectra (Figure 2E). Furthermore, according to unrestrained $1 \mu \mathrm{s}$ MD simulations in explicit water, non-glycosylated peptide 2 exhibits a random coil conformation in solution (Figure S3), which is different from the structure adopted by $\mathbf{2}^{*}$. Thus, despite the larger distance between the carbohydrate and the peptide backbone in glycopeptides $\mathbf{2}^{*}$ and $\mathbf{3}^{*}$, our results suggest that the sugar moiety still plays a role as a structural modulator, which presumably may reduce the conformational space accessible to the peptide backbone. Overall, unnatural glycopeptides $\mathbf{2}^{*}$ and $\mathbf{3}^{\star}$ display markedly different conformations in solution relative to the naturally occurring counterpart $1^{*}$ that are induced by the replacement of a single-atom in these compounds $(\mathrm{O} \rightarrow \mathrm{S} / \mathrm{Se})$. In particular, the conformational preference both at the glycosidic linkage and the unnatural residue (SThr4 or SeThr4) are shifted towards those of $1^{*}$ bound to an antiMUC1 monoclonal antibody. ${ }^{19}$ Thus, the energy cost associated to a conformational change of the glycopeptide from extended in solution to folded in the bound state is expected to be minimized (vide infra).

\section{Conformational analysis of the unnatural glycopeptides $2^{*}$ and $3^{*}$ bound to SCFV-SM3}

Crystals suitable for $X$-ray diffraction analysis of a recombinantly expressed single-chain variable fragment of the SM3 antibody (scFv-SM3) complexed with $\mathbf{2}^{*}$ and $\mathbf{3}^{*}$ were obtained. The $X$-ray structure of these complexes, solved at high resolution $(<2.0 \AA$, Table S2 and Figure 3, and Figure S7, PDB IDs: 5N7B and 6FRJ) revealed that the conformation of the bound peptide was nearly identical to that adopted by $1^{*}$ when bound to scFv-SM3 (Figure 3C). This result demonstrates that the antibody recognizes a welldefined epitope conformation, regardless of the nature of the glycosylated amino acid, characterized by torsion angles at the glycosylated residue typical of folded structures $(\phi$ and $\psi$ close to $-88^{\circ}$ and $10^{\circ}$, respectively). As detailed above, this conformation is also adopted in solution by the peptide backbone of glycopeptides $2^{*}$ and $3^{*}$ (Figure 2D and Figures S4 and S5). As for glycopeptide $1^{*}$, the stabilizing contacts in complexes $\mathbf{2}^{*} / \mathrm{scFv}-\mathrm{SM} 3$ and $\mathbf{3}^{*} / \mathrm{scFv}-\mathrm{SM} 3$ involve several hydrogen bonds, some of which are mediated by water molecules, as well as several stacking interactions (Figures $3 \mathrm{~A}$ and 3B).

Of note, two distinct binding modes are observed for glycopeptide $2^{*}$ in complex with scFv-SM3 that differ solely in the geometry of the glycosidic linkage. Binding mode $A$ is 
characterized by a glycosidic linkage with $\phi / \psi=87^{\circ} / 74^{\circ}$. This conformer corresponds to the structure adopted by $\mathbf{2}^{*}$ in solution (Figures $2 \mathrm{C}$ and $3 \mathrm{~A}$ ). Alternatively, in binding mode $B$, with glycosidic linkage angles $\phi / \psi \approx 90^{\circ} /-90^{\circ}$, the glycopeptide structure is stabilized by an intramolecular hydrogen bond between the $\mathrm{NH}$ of SThr4 and the endocyclic oxygen (O5) of GalNAc (Figure 3A). This binding mode was also found for the serine and a cysteine variants of the immunodominant PDTRP region of MUC1. ${ }^{19}$ Although binding mode A allows the $N$-acetyl group of GalNAc to stack with the aromatic ring of a tryptophan residue (Trp33H) of scFv-SM3, mode B impedes any direct contact between the sugar and the antibody. The electron density observed for the GalNAc moiety of glycopeptide $3^{*}$ is rather weak, which may suggest the simultaneous presence of both binding modes observed for derivative 2* (Figure 3B and Figure S7). Extensive MD simulations performed on the $\mathbf{2}^{\star} / \mathrm{scFv}-\mathrm{SM} 3$ complex supported that both binding modes ( $A$ and $B$ ) are stable in solution (Figures S8 and S9).

Interestingly, quantum mechanical (QM) calculations performed on abbreviated models of glycopeptides $\mathbf{1}^{*}$ and 2* (compounds 1' and 2', respectively; see Tables S3 and S4 and Figure S10) indicate that the larger repulsion between the $\beta$-methyl group of Thr and $\mathrm{H} 1$ of GalNAc in glycopeptide $\mathbf{1}^{*}$-as a result of the smaller size of the oxygen atomtogether with the more distorted geometry of the intramolecular hydrogen bond $\mathrm{O5}$ (GalNAc) and $\mathrm{NH}$ (Thr), leads to the lack of binding mode $\mathrm{B}$ in the natural occurring glycopeptide. 


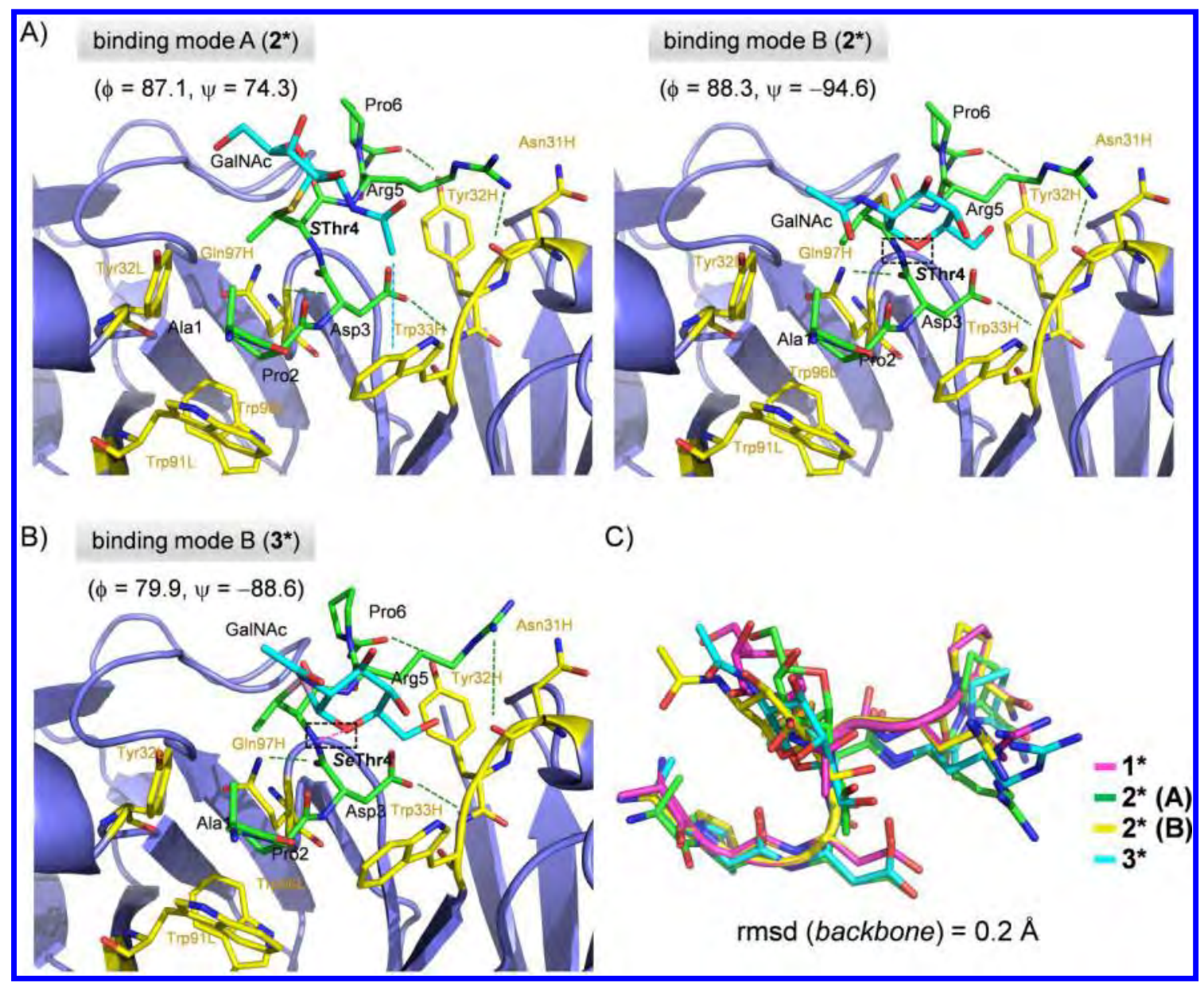

Figure 3. X-ray structures of glycopeptides $2^{*}(A)$ and $3^{*}(B)$ bound to the scFv-SM3 antibody (PDB IDs: 5N7B and 6FRJ). Glycopeptides carbon atoms are shown in green. Carbon atoms of key residues of ScFv-SM3 are colored yellow. Green dashed lines indicate hydrogen bonds between peptide backbones and ScFv-SM3 antibody. Pink dashed lines indicate the hydrogen bond between the $\mathrm{NH}$ of SThr (or SeThr) and 05 (see dashed boxes). Blue dashed line indicates a $\mathrm{CH} / \pi$ interaction between the $\mathrm{N}$-acetyl group of GalNAc and Trp33H in binding mode A. Note that the density corresponding to the GalNAc moiety in glycopeptide $3^{*}$ is only partial (Figure S7), strongly suggesting the existence of local flexibility. (C) Superposition of glycopeptides $1^{*}, \mathbf{2}^{*}$ and $\mathbf{3}^{*}$ in complex to the ScFv-SM3 antibody, which shows that the antibody recognizes the same conformation for the peptide backbone, regardless of the nature of the glycosylated residue.

\section{Affinity of the unnatural glycopeptides $2^{*}$ and $3^{*}$ towards ScFv-SM3}

Detailed conformational analysis of glycopeptides $2^{\star}$ and $3^{*}$ both in solution and bound to scFv-SM3 in comparison to that assumed in solution indicates that the structure of these peptides is preorganized for binding, which this is not the case for $1^{\star}$. Accordingly, 
a tighter binding would be expected for the unnatural derivatives (vide supra). To confirm this hypothesis, their binding affinities $\left(\mathrm{K}_{\mathrm{D}}\right)$ to ScFv-SM3 were measured by using surface plasmon resonance (SPR) assays (Figure 4 and Figures S11-S15). The highest affinities were observed for the unnatural glycopeptides $2^{*}$ and $3^{*}$ (with $K_{D}=168$ and $193 \mu \mathrm{M}$ at $25 \stackrel{\circ}{\circ}$, respectively). Notably, an improved affinity ( 20 -fold) was obtained relative to unglycosylated epitope 1 . The variation in the affinity of natural glycopeptide $1^{\star}$ with temperature is higher than for the unnatural counterparts. This result may indicate the existence of an extra entropic penalty associated with the binding of $\mathbf{1}^{*}$ (Figure 4B) and highlights in this respect the inherently different conformational behavior of unnatural glycopeptides $\mathbf{2}^{*}$ and $\mathbf{3}^{*}$, as already concluded through NMR experiments and MD simulations.

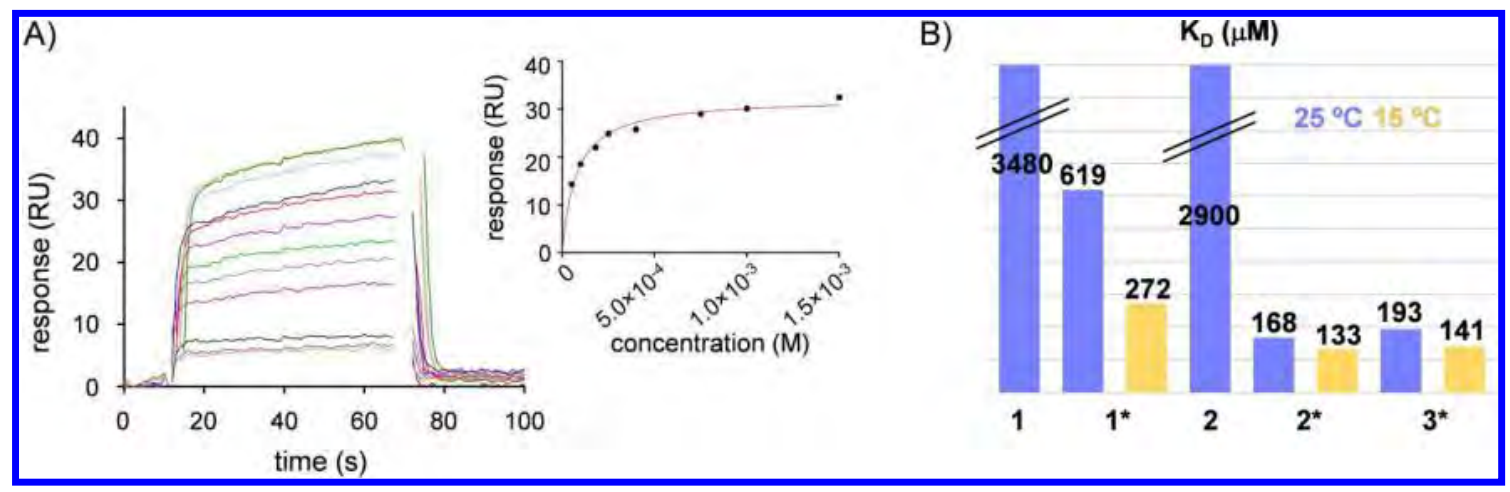

Figure 4. (A) SPR curves and response-concentration fit obtained for the binding of $\mathbf{2}^{\text {* }}$ towards scFv-SM3. (B) $K_{D}$ constants derived from SPR experiments for the studied (glyco)peptides.

\section{Preparation and in vivo studies of an anticancer vaccine based on an engineered glycopeptide.}

As discussed above, partially glycosylated peptides with sequences derived from MUC1 are an exciting niche of research for the development of therapeutic anticancer vaccines. As yet, none of them have so far succeeded in clinical trials, underlining the difficulty of inducing effective and durable immunological responses to a self-antigen such as tumorassociated-MUC1. ${ }^{12}$ Additional research is needed to understand how to break tolerance to self-antigens, which includes knowledge on how vaccine formulation are processed by the immune system and (glyco)peptide antigens are presented by the major histocompatibility complex (MHC) I and II. ${ }^{35-38}$

The results presented in this work prove that a single atom substitution at the glycosidic linkage has a remarkable impact on the structure of the glycopeptide in solution, especially at the glycosylated residue, which in turn may significantly affect peptide presentation and overall vaccine efficacy. Additionally, natural glycopeptides may suffer 
degradation from endogenous glycosidases, ${ }^{39,40}$ which in turn alters their effectiveness as immunizing antigens, ${ }^{41}$ while $S$-glycoside analogs have improved stability. ${ }^{42,43}$ These two considerations prompted us to test whether structurally engineered glycopeptide 12 could be used as tumor-associated antigen mimic through a nanoparticle-based immunogenic formulation (Figure 5A). Glycopeptide 12 comprises the complete tandem repeat sequence of MUC1 and features the SThr* residue described above. Additionally, this glycopeptide displays a (4S)-4-fluoro-L-proline (fPro) residue that replaces the Pro moiety at the beginning of the PDTRP epitope sequence. The motivation to select this doubly engineered glycopeptide was to combine the entropic benefit induced by SThr* by preorganizing the epitope structure for optimal binding, and the beneficial enthalpic effect produced by the fPro by enhancing antigen-antibody interactions. ${ }^{13}$ Moreover, one of us has been previously shown that PEGylated AuNPs could be used as efficient antigen carriers to establish humoral immunity against the tumor-associated form of MUC1 in mice, and the elicited antibodies did recognize the natural antigen on human breast cancer cells. ${ }^{44}$ These promising results led us to conjugate the MUC1 antigen mimic glycopeptide 12 with AuNPs in accordance with the strategy previously described (AuNP-12, Figure 5A, Figure S16 and Table S5). ${ }^{44}$ In this occasion, the synthetic effort was greatly reduced by omitting the extension of the glycopeptide with a CD4 T-cell peptide epitope and the immunogenic formulation was administered to the mice without any additional adjuvant.

The success of the conjugation reactions was easily confirmed through gel electrophoresis analysis, in which the conjugated AuNP-12 is characterized by a reduced electrophoretic mobility relative to the precursor, linker-functionalized AuNPs (Figure 5B). Additionally, a significant increase in hydrodynamic diameter was observed with dynamic light scattering (DLS, Table S5) upon conjugation. Peptide loading was determined by amino acid analysis to be 200 glycopeptides/AuNP.

Next, a standard immunization strategy was followed to test in vivo the immunogenic potential of AuNP-12. Thus, a group of five balb/c mice were immunized with a prime dose followed by three equal booster doses of AuNP-12 (each dose corresponds to 2 $\mu \mathrm{g}$ of the glycopeptide) at 21-days intervals whereas a control group was treated with phosphate buffered saline (PBS) as shown in Figure 5B. A week after the last booster dose, the mice were sacrificed, and the serum harvested. Analysis of the antisera showed that AuNP-12 can elicit a significant anti-MUC1 IgG antibody response. The total antibody endpoint titers (Figure S18) were better than those observed for the previously reported AuNP-based vaccine candidate in the presence of complete Freund's adjuvant. ${ }^{44}$ This result demonstrates that this adjuvant is fully dispensable for administration of our AuNP-based vaccine candidate, which are therefore self-adjuvating 
in their own right. Non-negligible IgM titers were also observed (although these were significantly lower than IgG titers), which suggests that glycopeptide 12 on AuNPs can induce class-switch recombination even without the use of a "universal" CD4 T-cell peptide. Next, the antibody isotypes in the antisera were evaluated. IgG1 was the predominant antibody in all antisera (Figure $5 \mathrm{C}$ ), which suggests that Th2 type immune responses were predominantly induced by AuNP-12 in these mice. Finally, IgG2a, IgG2b and carbohydrate-related IgG3 antibodies ${ }^{45}$ were detected in all animals albeit weakly.

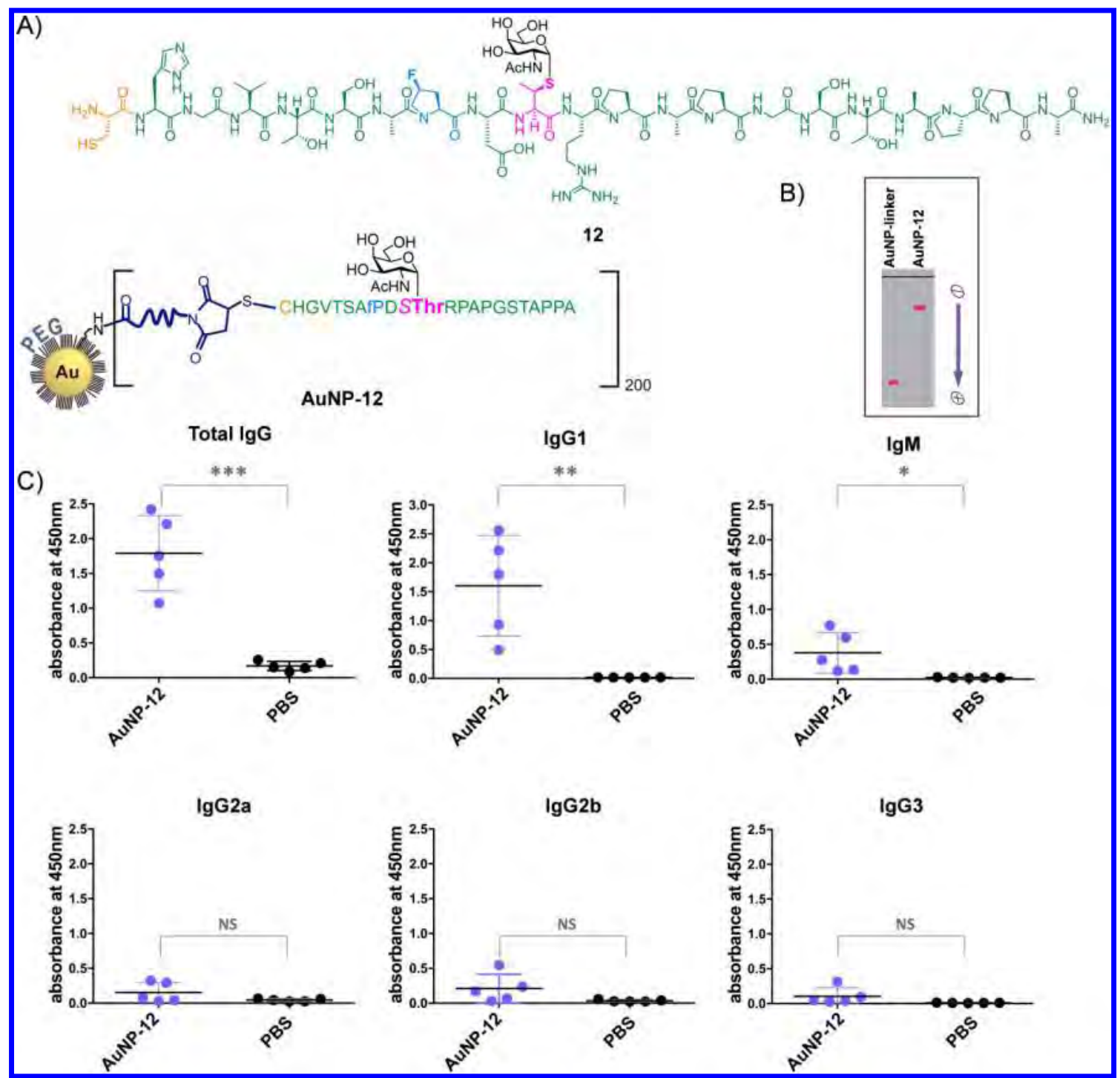

Figure 5. (A) Schematic representation of the vaccine candidate containing the engineered MUC1-like glycopeptide 12 attached to the surface of gold nanoparticles (AuNP-12). (B) Agarose gel electrophoresis of AuNP-linker (loaded with the SM(PEG) ${ }_{2}^{-}$ linker. See Supporting Information) and AuNP-12. (C) Total and sub-typing (IgG1, $\lg$ G2a/b, IgG3 and IgM) anti-MUC1 antibodies after immunizing mice $(n=5)$ with AuNP12. The ELISA plates were coated with glycopeptide 12 conjugated to bovine serum 
albumin. Horizontal lines indicate the mean for the group of 5 mice. Asterisks indicate statistically significant difference $\left({ }^{* * *} P<0.005\right.$, ${ }^{* *} P<0.01$, $\left.{ }^{*} P<0.05\right)$ and NS indicates no significant difference.

To confirm that the elicited antibodies were able to recognize selectively the native tumor-associated MUC1 antigen on human cancer cells, two human cancer cell lines (MCF-7, T47D) and the human embryonic kidney cell line (HEK293T) were stained with the mice antisera and analyzed by flow cytometry (Figure 6A). Indeed, the antisera reacted strongly with MCF-7 and T47D cells, which express tumor-associated MUC1 on their surface. Conversely, negligible low binding was observed for HEK293T cells, which is consistent with the lack of MUC1 on their surface. These results are in good agreement with those obtained from confocal microscopy (Figure 6B) that show the presence of the MUC1 antigen on the surface of MCF-7 and T47D cells (green color), but not on HEK293T cells. Notably, the antisera also positively stained cancer cells from biopsies of breast-cancer patients (right panel in Figure 6C and Figure S19), but no staining is observed in case of cells from healthy patients. Thus, these results demonstrate the antigen mimic potential of unnatural glycopeptide 12. 


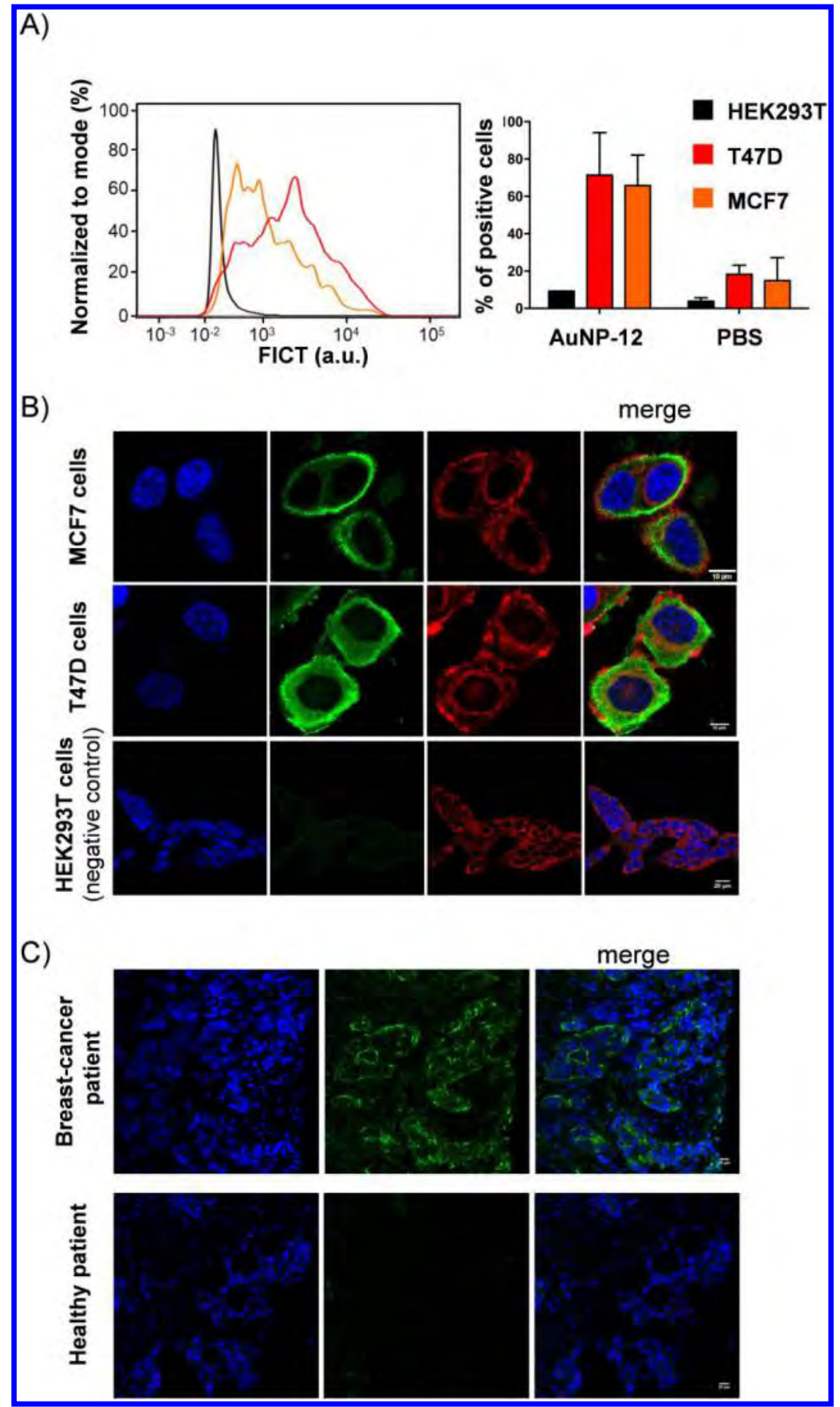

Figure 6. (A) Staining of living cells with the antisera of mice immunized with AuNP-12 analyzed by flow cytometry: HEK293T (black line), MCF7 (orange line) and T47D (red line). Staining with 1:100 dilution of sera and visualization with a mouse secondary $\alpha-$ lgG-488 antibody. (B) Confocal microscopy images show that mice antisera after vaccination with AuNP-12 do not stain HEK293T cells as expected because these cells do not express tumor-associated MUC1 on their surface. On the contrary, breast cancer cells MCF7 and T47D expressing tumor-associated MUC1 are positively stained by mice antisera. Blue = Hoechst (nuclei); Green = secondary anti-mouse IgG Alexa 488 (tumorassociated MUC1); Red = CellMask Deep Red (membrane dye). (C) The antisera of 
mice vaccinated with AuNP-12, positively stain tissue biopsies from breast-cancer patients. Blue = Hoechst (nuclei); Green = secondary anti-mouse IgG Alexa 488 (tumorassociated MUC1).

\section{Conclusions}

Our experimental evidence strongly suggests that it is possible to fine-tune the conformational preferences of GalNAc-containing glycopeptides in solution by employing a simple oxygen-for-sulfur or oxygen-for-selenium substitution at the glycosidic linkage. These simple chemical modifications have a significant structural impact allowing the peptide backbone to adopt a preorganized structure that is optimally suited for antibody binding, as confirmed by the improved binding affinity to a model antiMUC1 antibody. Additionally, the potential of a dually modified glycopeptide (fPro and SThr) as tumor-associated MUC1 antigen mimic has been demonstrated in vivo. Significantly, the antisera of mice vaccinated with AuNP-12 recognize cancer cells with high selectivity in biopsies of breast-cancer patients. This result confirms that the antibodies generated against the engineered antigen are able to recognize the naturally occurring antigen in its physiological context. Finally, we envision the strategy presented here to be of general interest because it may be applied to modulate the affinity of biologically relevant glycopeptides towards their receptors.

\section{Acknowledgements}

We thank the Ministerio de Economía y Competitividad (projects CTQ2015-67727-R, CTQ2013-44367-C2-2-P, CTQ2015-64597-C2-1P, CTQ2017-89750-R, CTQ201790088-R, CTQ2015-70524-R, and BFU2016-75633-P) and the Italian Ministry of Education, University and Research (MIUR) (PRIN 2015 contract nr. 2015RNWJAM). I.C. thanks Universidad de La Rioja for a FPI grant. A.G. and G.J.L.B. thank FCT Portugal (PhD studentship, SFRH/BD/115932/2016, and FCT Investigator IF/00624/2015, respectively). G.J.L.B. holds a Royal Society URF (UF110046 and URF $\backslash R \backslash 180019$ ) and an ERC StG (Taglt, GA No. 676832). F.C. and G. J. L. B thank the EU (Marie-Sklodowska Curie ITN, Protein Conjugates, GA No. 675007). O.B. and G.J.O. are Ramón y Cajal Fellows (RYC-2015-17705 and RYC-2013-14706, respectively). R.H-G. thanks Agencia Aragonesa para la Investigación y Desarrollo (ARAID) and the Diputación General de Aragón (DGA, E34_R17) for financial support. The research that led to these results also attracted funding from the FP7 (2007-2013) under BioStruct-X (grant agreement $\mathrm{N}^{\circ} 283570$ and BIOSTRUCTX_5186). We also thank the ALBA synchrotron radiation source; in particular the beamline XALOC facility. We thank Emanuele Papini and Regina Tavano (University of Padua, Italy) for insightful 
discussions about the immunogenicity of glycopeptide-functionalized AuNPs, and Vito Maggi and Valentina Rosato (IIT-Lecce) for expert technical assistance. Computational resources of CESGA (Santiago de Compostela) and BERONIA (Universidad de La Rioja) were used in this work. The authors thank Vikki Cantrill for her help with the preparation and editing of this manuscript.

\section{Competing financial interests}

The authors declare no competing financial interests.

\section{Supporting Information}

Synthesis and characterization of the glycopeptides $2^{*}$ and $3^{*}$ and the AuNP-based vaccine, conformational analysis in solution of glycopeptides $2^{*}$ and $3^{\star}$, details of the $X$ ray structure of $2^{*}$ and $3^{*}$ bound to ScFv-SM3, SPR curves and response-concentration fit obtained for the binding of the (glyco)peptides, cartesian coordinates, electronic energies, Gibbs free energies and lowest frequencies of the DFT calculated structures, additional molecular dynamics simulations figures, immunization protocol, antibodies titers and antibody isotypes, antibodies reactivity towards human cancer cell lines determined by flow cytometry analysis and analyzed by confocal microscopy, and studies on cancer cell from breast-cancer patients. This material is available free of charge via the Internet at http://pubs.acs.org

\section{References}

(1) Hollingsworth, M. A.; Swanson, B. J. Mucins in Cancer: Protection and Control of the Cell Surface. Nat. Rev. Cancer 2004, 4, 45-60.

(2) Kufe, D. W. Mucins in Cancer: Function, Prognosis and Therapy. Nat. Rev. Cancer 2009, 9, 874-885.

(3) Senapati, S.; Das, S.; Batra, S. K. Mucin-Interacting Proteins: From Function to Therapeutics. Trends Biochem. Sci. 2010, 35, 236-245.

(4) Taylor-Papadimitriou, J.; Burchell, J. M. Mucins and Cancer, TaylorPapadimitriou, J., Burchell, J. M., Eds.; Future Medicine: London, 2013.

(5) Ju, T.; Otto, V. I.; Cummings, R. D. The Tn Antigen-Structural Simplicity and Biological Complexity. Angew. Chem. Int. Ed. 2011, 50, 1770-1791.

(6) Schuman, J.; Campbell, A. P.; Koganty, R. R.; Longenecker, B. M. Probing the Conformational and Dynamical Effects of O-Glycosylation Within the Immunodominant Region of a MUC1 Peptide Tumor Antigen. J. Pept. Res. 2003, 61, 91-108. 
(7) Karsten, U.; Serttas, N.; Paulsen, H.; Danielczyk, A.; Goletz, S. Binding Patterns of DTR-Specific Antibodies Reveal a Glycosylation-Conditioned Tumor-Specific Epitope of the Epithelial Mucin (MUC1). Glycobiology 2004, 14, 681-692.

(8) Wilson, R. M.; Danishefsky, S. J. A Vision for Vaccines Built from Fully Synthetic Tumor-Associated Antigens: From the Laboratory to the Clinic. J. Am. Chem. Soc. 2013, 135, 14462-14472.

(9) Buskas, T.; Thompson, P.; Boons, G.-J. Immunotherapy for Cancer: Synthetic Carbohydrate-Based Vaccines. Chem. Commun. 2009, 105, 5335-5349.

(10) Martínez-Sáez, N.; Peregrina, J. M.; Corzana, F. Principles of Mucin Structure: Implications for the Rational Design of Cancer Vaccines Derived from MUC1Glycopeptides. Chem. Soc. Rev. 2017, 46, 7154-7175.

(11) Gaidzik, N.; Westerlind, U.; Kunz, H. The Development of Synthetic Antitumour Vaccines from Mucin Glycopeptide Antigens. Chem. Soc. Rev. 2013, 42, 44214442.

(12) Hossain, M. K.; Wall, K. A. Immunological Evaluation of Recent MUC1 Glycopeptide Cancer Vaccines. Vaccines 2016, 4, 25-13.

(13) Somovilla, V. J.; Bermejo, I. A.; Albuquerque, I. S.; Martínez-Sáez, N.; CastroLópez, J.; Garcia-Martin, F.; Compañón, I.; Hinou, H.; Nishimura, S.-I.; JiménezBarbero, J.; Asensio, J. L.; Avenoza, A.; Busto, J. H.; Hurtado-Guerrero, R.; Peregrina, J. M.; Bernardes, G. J. L.; Corzana, F. The Use of Fluoroproline in MUC1 Antigen Enables Efficient Detection of Antibodies in Patients with Prostate Cancer. J. Am. Chem. Soc. 2017, 139, 18255-18261.

(14) Coltart, D. M.; Royyuru, A. K.; Williams, L. J.; Glunz, P. W.; Sames, D.; Kuduk, S. D.; Schwarz, J. B.; Chen, X.-T.; Danishefsky, S. J.; Live, D. H. Principles of Mucin Architecture: Structural Studies on Synthetic Glycopeptides Bearing Clustered Mono-, Di-, Tri-, and Hexasaccharide Glycodomains. J. Am. Chem. Soc. 2002, 124, 9833-9844.

(15) Corzana, F.; Busto, J. H.; Jiménez-Oses, G.; Asensio, J. L.; Jiménez-Barbero, J.; Peregrina, J. M.; Avenoza, A. New Insights into $\alpha$-GalNAc-Ser Motif: Influence of Hydrogen Bonding versus Solvent Interactions on the Preferred Conformation. J. Am. Chem. Soc. 2006, 128, 14640-14648.

(16) Corzana, F.; Busto, J. H.; Jiménez-Oses, G.; García de Luis, M.; Asensio, J. L.; Jiménez-Barbero, J.; Peregrina, J. M.; Avenoza, A. Serine Versus Threonine Glycosylation: The Methyl Group Causes a Drastic Alteration on the Carbohydrate Orientation and on the Surrounding Water Shell. J. Am. Chem. Soc. 2007, 129, 9458-9467. 
(17) Bermejo, I. A.; Usabiaga, I.; Compañón, I.; Castro-López, J.; Insausti, A.; Fernández, J. A.; Avenoza, A.; Busto, J. H.; Jiménez-Barbero, J.; Asensio, J. L.; Peregrina, J. M.; Jiménez-Oses, G.; Hurtado-Guerrero, R.; Cocinero, E. J.; Corzana, F. Water Sculpts the Distinctive Shapes and Dynamics of the TumorAssociated Carbohydrate Tn Antigens: Implications for Their Molecular Recognition. J.Am. Chem. Soc. 2018, 140, 9952-9960.

(18) Burchell, J.; Gendler, S.; Taylor-Papadimitriou, J.; Girling, A.; Lewis, A.; Millis, R.; Lamport, D. Development and Characterization of Breast Cancer Reactive Monoclonal Antibodies Directed to the Core Protein of the Human Milk Mucin. Cancer Res. 1987, 47, 5476-5482.

Martínez-Sáez, N.; Castro-López, J.; Valero-Gónzalez, J.; Madariaga, D.; Compañón, I.; Somovilla, V. J.; Salvadó, M.; Asensio, J. L.; Jiménez-Barbero, J.; Avenoza, A.; Busto, J. H.; Bernardes, G. J. L.; Peregrina, J. M.; HurtadoGuerrero, R.; Corzana, F. Deciphering the Non-Equivalence of Serine and Threonine O-Glycosylation Points: Implications for Molecular Recognition of the Tn Antigen by an anti-MUC1 Antibody. Angew. Chem. Int. Ed. 2015, 54, 98309834.

(20) Asensio, J. L.; Ardá, A.; Cañada, F. J.; Jiménez-Barbero, J. CarbohydrateAromatic Interactions. Acc. Chem. Res. 2013, 46, 946-954.

(21) Jiménez-Moreno, E.; Gómez, A. M.; Bastida, A.; Corzana, F.; Jiménez-Oses, G.; Jiménez-Barbero, J.; Asensio, J. L. Modulating Weak Interactions for Molecular Recognition: A Dynamic Combinatorial Analysis for Assessing the Contribution of Electrostatics to the Stability of $\mathrm{CH} / \pi$ Bonds in Water. Angew. Chem.Int.Ed. 2015, 54, 4344-4348.

(22) García-Herrero, A.; Montero, E.; Muñoz, J. L.; Espinosa, J. F.; Vián, A.; García, J. L.; Asensio, J. L.; Cañada, F. J.; Jiménez-Barbero, J. Conformational Selection of Glycomimetics at Enzyme Catalytic Sites: Experimental Demonstration of the Binding of Distinct High-Energy Distorted Conformations of C-, S-, and O-Glycosides by E. Coli $\beta$-Galactosidases. J. Am. Chem. Soc. 2002, 124, 4804-4810.

(23) Dziadek, S.; Griesinger, C.; Kunz, H.; Reinscheid, U. M. Synthesis and Structural Model of an $\alpha(2,6)$-Sialyl-T Glycosylated MUC1 Eicosapeptide under Physiological Conditions. Chem. Eur. J. 2006, 12, 4981-4993.

(24) Gracia-Vitoria, J.; Osante, I.; Cativiela, C. Stereoselective Synthesis of Modified Cysteines. Tetrahedron:Asvmmetrv 2017, 28, 215-245. 
(25) Chen, S.; Fahmi, N. E.; Nangreave, R. C.; Mehellou, Y.; Hecht, S. M. Synthesis of pdCpAs and Transfer RNAs Activated with Thiothreonine and Derivatives. Bioorg. Med. Chem. 2012, 20, 2679-2689.

Narayan, R. S.; VanNieuwenhze, M. S. Versatile and Stereoselective Syntheses of Orthogonally Protected $\beta$-Methylcysteine and $\beta$-Methyllanthionine. Org. Lett. 2005, 7, 2655-2658.

(27) Jobron, L.; Hummel, G. Solid-Phase Synthesis of New S-Glycoamino Acid Building Blocks. Org. Lett. 2000, 2, 2265-2267.

(28) Dyson, H. J.; Wright, P. E. Defining Solution Conformations of Small Linear Peptides. Annu. Rev. Biophvs. Chem. 1991, 20, 519-538.

(29) Pearlman, D. A.; Kollman, P. A. Are Time-Averaged Restraints Necessary for Nuclear Magnetic Resonance Refinement? J. Mol. Biol. 1991, 220, 457-479.

(30) Pearlman, D. How Is an NMR Structure Best Defined? An Analysis of Molecular Dynamics Distance-Based Approaches. J. Biomol. NMR 1994, 4, 1-16.

(31) Fernández-Tejada, A.; Corzana, F.; Busto, J. H.; Jiménez-Osés, G.; JiménezBarbero, J.; Avenoza, A.; Peregrina, J. M. Insights into the Geometrical Features Underlying $\beta$-O-GlcNAc Glycosylation: Water Pockets Drastically Modulate the Interactions Between the Carbohydrate and the Peptide Backbone. Chem. Eur. J. 2009, 15, 7297-7301.

(32) Madariaga, D.; Martínez-Sáez, N.; Somovilla, V. J.; Coelho, H.; ValeroGónzalez, J.; Castro-López, J.; Asensio, J. L.; Jiménez-Barbero, J.; Busto, J. H.; Avenoza, A.; Marcelo, F.; Hurtado-Guerrero, R.; Corzana, F.; Peregrina, J. M. Detection of Tumor-Associated Glycopeptides by Lectins: the Peptide Context Modulates Carbohydrate Recognition. ACS Chem. Biol. 2015, 10, 747-756.

(33) Mazeau, K.; Tvaroska, I. PCILO Quantum-Mechanical Relaxed Conformational Energy Map of Methyl 4-Thio-A-Maltoside in Solution. Carbohydr. Res. 1992, 225, 27-41.

(34) Aydillo, C.; Compañón, I.; Avenoza, A.; Busto, J. H.; Corzana, F.; Peregrina, J. M.; Zurbano, M. M. S-Michael Additions to Chiral Dehydroalanines as an Entry to Glycosylated Cysteines and a Sulfa-Tn Antigen Mimic. J. Am. Chem. Soc. 2014, 136, 789-800.

(35) Lakshminarayanan, V.; Thompson, P.; Wolfert, M. A.; Buskas, T.; Bradley, J. M.; Pathangey, L. B.; Madsen, C. S.; Cohen, P. A.; Gendler, S. J.; Boons, G.-J. Immune Recognition of Tumor-Associated Mucin MUC1 Is Achieved by a Fully Synthetic Aberrantly Glycosylated MUC1 Tripartite Vaccine. Proc. Natl. Acad. Sci. U.S.A. 2012, 109, 261-266. 
(36) Lakshminarayanan, V.; Supekar, N. T.; Wei, J.; McCurry, D. B.; Dueck, A. C.; Kosiorek, H. E.; Trivedi, P. P.; Bradley, J. M.; Madsen, C. S.; Pathangey, L. B.; Hoelzinger, D. B.; Wolfert, M. A.; Boons, G.-J.; Cohen, P. A.; Gendler, S. J. MUC1 Vaccines, Comprised of Glycosylated or Non-Glycosylated Peptides or Tumor-Derived MUC1, Can Circumvent Immunoediting to Control Tumor Growth in MUC1 Transgenic Mice. PLoS ONE 2016, 11, e0145920.

(37) Wu, X.; Yin, Z.; McKay, C.; Pett, C.; Yu, J.; Schorlemer, M.; Gohl, T.; Sungsuwan, S.; Ramadan, S.; Baniel, C.; Allmon, A.; Das, R.; Westerlind, U.; Finn, M. G.; Huang, X. Protective Epitope Discovery and Design of MUC1-Based Vaccine for Effective Tumor Protections in Immunotolerant Mice. J. Am. Chem. Soc. 2018, 140, 16596-16609.

(38) Wolfert, M. A.; Boons, G.-J. Adaptive Immune Activation: Glycosylation Does Matter. Nat.Chem.Biol. 2013, 9, 776-784.

(39) Richichi, B.; Thomas, B.; Fiore, M.; Bosco, R.; Qureshi, H.; Nativi, C.; Renaudet, O.; BenMohamed, L. A Cancer Therapeutic Vaccine Based on Clustered TnAntigen Mimetics Induces Strong Antibody-Mediated Protective Immunity. Angew. Chem. Int. Ed. 2014, 53, 11917-11920.

(40) Nativi, C.; Renaudet, O. Recent Progress in Antitumoral Synthetic Vaccines. ACS Med. Chem. Lett. 2014, 5, 1176-1178.

(41) Rich, J. R.; Bundle, D. R. S-Linked Ganglioside Analogues for Use in Conjugate Vaccines. Ora.Lett. 2004, 6, 897-900.

(42) Huo, C.-X.; Zheng, X.-J.; Xiao, A.; Liu, C.-C.; Sun, S.; Lv, Z.; Ye, X.-S. Synthetic and Immunological Studies of N-Acyl Modified S-Linked STn Derivatives as Anticancer Vaccine Candidates. Org. Biomol. Chem. 2015, 13 (12), 3677-3690.

(43) Bousquet, E.; Spadaro, A.; Pappalardo, M. S.; Bernardini, R.; Romeo, R.; Panza, L.; Ronsisvalle, G. Synthesis and Immunostimulating Activity of a Thioglycolipopeptide Glycomimetic as a Potential Anticancer Vaccine Derived From Tn Antigen. J. Carbohyd. Chem. 2000, 19, 527-541.

(44) Cai, H.; Degliangeli, F.; Palitzsch, B.; Gerlitzki, B.; Kunz, H.; Schmitt, E.; Fiammengo, R.; Westerlind, U. Glycopeptide-Functionalized Gold Nanoparticles for Antibody Induction Against the Tumor Associated Mucin-1 Glycoprotein. Bioora. Med.Chem. 2016, 24, 1132-1135.

(45) Haji-Ghassemi, O.; Blackler, R. J.; Martin Young, N.; Evans, S. V. Antibody Recognition of Carbohydrate Epitopes. Glycobiology 2015, 25, 920-952. 
TOC

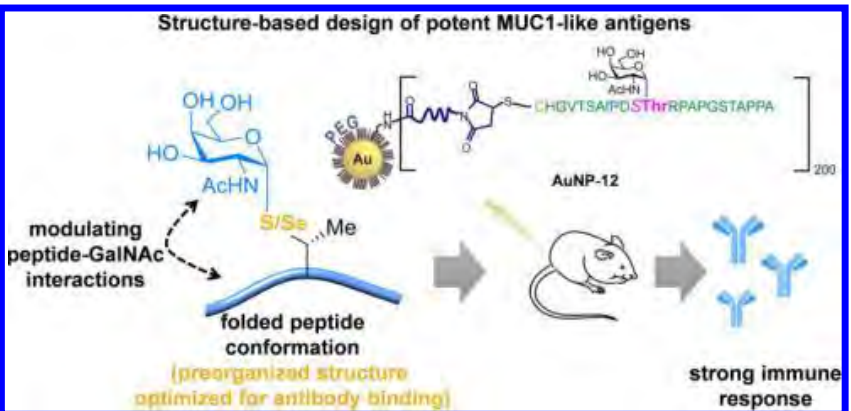

14

15

16

17 